\title{
1 Optimization of SARS-CoV-2 detection by RT-QPCR without RNA extraction
}

3 Natacha Merindol ${ }^{1,2}$, Geneviève Pépin ${ }^{3}$, Caroline Marchand ${ }^{2}$, Marylène Rheault ${ }^{2}$, Christine

4 Peterson $^{2}$, André Poirier $^{2,4}$, Hugo Germain ${ }^{1,2^{*}}$, Alexis Danylo ${ }^{2,4^{*}}$

$5 \quad$ * contributed equally to this work

6

$7 \quad{ }^{1}$ Département de chimie, biochimie et physique, Université du Québec à Trois-Rivières, Trois-

8 Rivières, Québec, Canada

$9{ }^{2}$ Centre Intégré Universitaire de santé et services sociaux de la Mauricie et Centre du Québec,

10 Trois-Rivières, Quebec, Canada

$11{ }^{3}$ Département de biologie médicale, Université du Québec à Trois-Rivières, Trois-Rivières,

12 Québec, Canada

$13{ }^{4}$ Département de microbiologie infectiologie et immunologie, faculté de médecine, Université

14 de Montréal, Montréal, Québec, Canada

15 NM: Natacha.Merindol@uqtr.ca

16 HG: Hugo.Germain@uqtr.ca

17 GP: Genevieve.Pepin3@uqtr.ca

18 CM: caroline_marchand_labo@ssss.gouv.qc.ca

19 MR: marylene_rheault@ssss.gouv.qc.ca

20 CP: christine_peterson@ssss.gouv.qc.ca

21 AP: andre_poirier_chrtr@ssss.gouv.qc.ca

22 Corresponding author: Alexis_Danylo@ssss.gouv.qc.ca 


\section{Abstract}

26 Rapid and reliable screening of SARS-CoV-2 is fundamental to assess viral spread and limit the

27 pandemic we are facing. In this study we evaluated the reliability and the efficiency of a direct

28 RT-QPCR method (without RNA extraction) using SeeGene Allplex ${ }^{\mathrm{TM}}$ 2019-nCoV RT-QPCR and

29 the influence of swab storage media composition on further viral detection.

30 We show that SeeGene's assay provides similar efficiency as the RealStar® SARS-CoV-2 RT-PCR

31 kit (Altona Diagnostics), and that RNA extraction is not necessary nor advantageous if samples

32 are stored in UTM or molecular water but is recommended if samples are stored in saline

33 solution and in Hanks medium.

35 Keywords: COVID19, SARS-CoV-2, coronavirus, virus detection, PCR, RNA extraction 


\section{Introduction}

46 The SARS-CoV-2 pandemic that the world is fighting since January 2020 has already caused

47 devastating mortality (1). As we are facing our biggest public health threat in the modern history,

48 there is a huge number of samples testing to be performed. Most of the current methods

49 described so far are based on viral genes detection by RT-QPCR from patients fluids, including

50 from the respiratory tract (2-4).

51 To slow the spread of the pandemic, screening is the key (5). This allows to isolate positive

52 patients and stop the transmission. Many medical laboratories now have automated protocol to

53 test the samples. These protocols are based on a Real-time PCR amplification on extracted RNA

54 from fluids or swabs stored in media. However, in many laboratories worldwide, fear of

55 shortage in the chain of supplies is growing. Shortage of RNA extraction kit, PCR detection kits

56 and even swabs storage media will likely arise in many testing laboratories.

57 This outstanding situation called for alternatives protocols to ensure the continuity of rapid

58 testing in medical laboratories. In addition, the development of new methods could also be

59 used in case of an increase in demand for testing. In this paper, we have compared the

60 concordance rate of positive viral genes detection of extracted RNA from the Abott M2000sp

61 automated plate preparation system using Altona RealStar ${ }^{\oplus}$ SARS-CoV-2 RT-PCR Kit RUO with

62 the SeeGene Allplex ${ }^{\mathrm{TM}}$ 2019-nCoV RT-QPCR Assay running on CFX96 BioRad. In addition, we

63 demonstrate that direct testing of viral genes by skipping the RNA extraction step on swabs

64 storage media could be achieved when the samples originate from standard UTM media and

65 from molecular water. 


\section{Methods}

69 Specimens were collected with nasopharyngeal and oropharyngeal swabs, and were stored in

70 different mediums. Mediums used were UTM $^{\circledR}$ (Remel RE12569), homemade Hanks medium,

71 Saline and molecular grade $\mathrm{H} 2 \mathrm{O}$.

72 Oro-nasopharyngeal swabs were inactivated at $95^{\circ} \mathrm{C}$ for $5 \mathrm{~min}$ and stored at $4^{\circ} \mathrm{C}$ or $-80^{\circ} \mathrm{C}$. RNA

73 was extracted from $700 \mu \mathrm{l}$ of $2 \mathrm{ml}$ of patient's swab medium using Abbott $m$ Sample Preparation

74 Systems DNA kit on m2000sp instrument; the eluate was $90 \mu \mathrm{l}$. RT-QPCR plates were automated:

75 prepared on the Abbott m2000sp and routinely detected on m2000rt using the Altona

76 RealStar ${ }^{\oplus}$ SARS-CoV-2 RT-PCR Kit RUO according to the manufacturer's instruction. 10 $\mu$ of RNA

77 was used in $20 \mu l$.

78 SeeGene Allplex ${ }^{\mathrm{TM}}$ 2019-nCoV RT-QPCR Assay was manually performed on a CFX96 BioRad

79 machine according to the manufacturer's instruction. $7 \mu$ of RNA or patient' swab medium was

80 used in a total of $25 \mu$ and 45 cycles of amplification were performed unless specified otherwise.

81 A patient was considered positive when a positive signal (gene $\mathrm{E}$ or $\mathrm{S}$ in Altona, genes $\mathrm{E}, \mathrm{N}$ or

82 RdRP in SeeGene) was detected at any Cts. A patient was considered negative if the internal

83 control was amplified but not the viral genes (N, E, S and RdRP). 


\section{Following RNA extraction and RT-QPCR SeeGene vs Altona}

90 To determine the concordance between the standard Altona method and the SeeGene protocol,

91 RNA extracted from 65 SARS-CoV-2-positive and 23 -negative samples were tested using

92 SeeGene kit in three consecutive assays. 23 negative samples were confirmed negative, 64 out

93 of 65 positive samples were confirmed positive $(96,92 \%)$. One sample, detected at $\mathrm{Ct}=35.6$

94 (gene S only) with Altona's kit was missed using SeeGene's kit (Figure 1, Tables 1 and 2). Ct

95 means between the two methods cannot be directly compared as SeeGene uses less RNA,

96 which can have important and this assay was performed following an additional cycle of

97 freeze/thaw. Moreover, both manufacturers target different genes and likely do not use the

98 same primers for the E gene in the PCR which can also results in Ct variation. Although both

99 manufacturers neither use the same amount of RNA in the RT-QPCR reaction nor detect the

100 same viral genes, all compounding factors which could lead to important differences in Ct

101 between the two methods, we still observed a strong correlation between the Ct values

102 obtained by both methods ( $r=0.9746 ; p<0.0001)$.

104 RT-QPCR directly from swabs: without RNA extraction

105 The swab storage media for respiratory virus detection can have important impact on the RT-

106 PCR efficiency, when this media is used directly for PCR reaction, without RNA extraction. To

107 assess the feasibility of direct RT-QPCR using different media we performed the following

108 experiments.

109

110

Samples stored in UTM 
11117 samples stored in UTM media, previously assessed as positive by the Altona method with

112 RNA extraction, were used for direct RT-QPCR in two independent experiments. 100\% were

113 detected using SeeGene (gene N) and Ct means were equivalent whether or not RNA was

114 extracted before the RT-QPCR (Figure 2; Table 1 and 2). This experiment was also repeated in

$115 \quad 12.5 \mu \mathrm{l}$ of final volume and similar results were obtained.

117 Samples stored in Hanks

118 Due to a shortage of UTM media, swabs were stored in Hanks medium and analysed for direct

119 RT-QPCR. 94 samples previously assessed as positive by the Altona method (with RNA extraction)

120 stored in Hanks medium were used for direct RT-QPCR in two independent experiments. 21

$121(2,3 \%)$ reactions did not show amplification neither of viral genes nor of the internal control

122 suggesting RT-QPCR inhibition. 75,53\% ( $n=71)$ were positive following analysis (Tables 1 and 2,

123 Figure 3). The addition of 5 cycles (from 45 cycles to 50 cycles) for 64 of the samples helped with

124 the detection of 5 samples that showed delayed Cts.

125 Ct values cannot be directly compared to Altona's, as less volume of sample was added in the

126 mastermix, the assays were performed following an additional cycle of freeze/thaw, and the

127 target genes and primers used by both manufacturers are not the same.

129 Samples stored in water

130 To determine if molecular grade water could be used as swabs storage media without impacting

131 direct RT-QPCR, three positive swabs were stored in water at $4^{\circ} \mathrm{C}$. Eight serial dilutions (1:10)

132 were performed on two of these samples. From the first patient, the undiluted sample and 4 of 
1337 dilutions showed amplification of SARS-CoV-2 N gene and S gene using RNA with both Altona

134 and SeeGene, respectively. In this same sample comparable amounts of viral $\mathrm{N}$ gene was

135 detected without RNA extraction using SeeGene (Figure 4). Cts can be compared between the

136 two methods in this case as there was no additional Freeze/thaw cycle for this experiment. This

137 experiment was confirmed using $12 \mu \mathrm{l}$ as sample volume in the mastermix. An addition of 5

138 other patients were efficiently amplified (Figure 5).

139 In the second sample, SARS-CoV-2 could be detected only from the RNA extracted in the

140 undiluted specimen using Altona's kit at $\mathrm{Ct}=35,6$. The experiment was repeated using SeeGene

141 and SARS-CoV-2 $\mathrm{N}$ gene was detected from $\mathrm{H} 2 \mathrm{O}$ (stored at $4^{\circ} \mathrm{C}$ for 24 hours) at $\mathrm{Ct}=37.1$ but not

142 from extracted RNA (Table 1 and 2).

143

144 Samples stored in saline solution

145 To determine if saline solution could be used as swabs storage media without impacting direct

146 RT-PCR, five RNA eluate from samples with high Cts (between 12,61 and 14,22) following

147 Altona's amplification were diluted in water (1:100) and in saline solution (1:100). Dilutions

148 were directly used for RT-QPCR. As previously observed, amplification could be reliably detected

149 in samples stored in water. However, when samples were stored in saline water, a loss of 10 Cts

150 for the N gene was measured following SeeGene RT-QPCR and the E gene could not be

151 amplified despite 50 cycles.

152

153

154 Stability of long-term storage of swabs in water at $4^{\circ} \mathrm{C}$ 
155 Centralization and increased number of screenings may lead to swabs storage at $4^{\circ} \mathrm{C}$ for several

156 days. To assess viral RNA stability at $4^{\circ} \mathrm{C}$, we quantified levels of N, E and RDRP genes directly

157 from a swab following four $1: 10$ serial dilutions stored for $0,24,72$ hours and for 5 days at $4^{\circ} \mathrm{C}$.

158 RNase inhibitor was added to the patient swab and serial dilutions in molecular grade water

159 were performed (hence the RNAse inhibitor was also diluted). Viral RNA was detected at 0, 24

160 and 72 hours at all dilutions but 1:10 000. Ct values were highly constant at all timepoints for

161 the three viral genes for undiluted, 1:10 and 1:100 diluted swabs (E gene, data not shown). The

162 addition of RNase inhibitor probably increased RNA stability of the undiluted swab.

163 Nevertheless, RNA remained highly stable even when diluted hundred times. Samples diluted

1641000 times were less accurately amplified: RDRP was detected up to $24 \mathrm{~h}$.

\section{Conclusion}

168 Our results suggest that: i) the SeeGene Allplex ${ }^{T M}$ 2019-nCoV RT-QPCR Assay provides similar

169 efficiency as the Altona RealStar ${ }^{\circledR}$ SARS-CoV-2 RT-PCR Kit RUO, ii) RNA extraction is not

170 necessary if samples are stored in UTM or molecular grade water; iii) RNA extraction is

171 recommended if samples are stored in saline water and in Hanks medium. The number of cycles

172 can be increased to 50 to detect low-producers.

\section{3}

174

175

176 References 
1. Coronaviridae Study Group of the International Committee on Taxonomy of V. The species Severe acute respiratory syndrome-related coronavirus: classifying 2019-nCoV and naming it SARS-CoV-2. Nat Microbiol. 2020;5(4):536-44.

2. Corman VM, Landt O, Kaiser M, Molenkamp R, Meijer A, Chu DKW, et al. Detection of 2019 novel coronavirus (2019-nCoV) by real-time RT-PCR. Euro Surveill. 2020;25(3).

1843 3. Jin YH, Cai L, Cheng ZS, Cheng H, Deng T, Fan YP, et al. A rapid advice guideline for the diagnosis and treatment of 2019 novel coronavirus (2019-nCoV) infected pneumonia (standard version). Mil Med Res. 2020;7(1):4.

187 4. Chan JF, Yip CC, To KK, Tang TH, Wong SC, Leung KH, et al. Improved molecular diagnosis of COVID-19 by the novel, highly sensitive and specific COVID-19-RdRp/Hel real-time reverse transcription-polymerase chain reaction assay validated in vitro and with clinical specimens. J

190 Clin Microbiol. 2020.

5. Tang YW, Schmitz JE, Persing DH, Stratton CW. The Laboratory Diagnosis of COVID-19 Infection: Current Issues and Challenges. J Clin Microbiol. 2020.

6. Lippi G, Simundic AM, Plebani M. Potential preanalytical and analytical vulnerabilities in the laboratory diagnosis of coronavirus disease 2019 (COVID-19). Clin Chem Lab Med. 2020.

\section{Figures legend}

201 Each dot represents 1 of 65 reaction, each corresponding to a patient. The grey dot represents

202 the only sample that was missed using SeeGene, detected at $\mathrm{Ct}=35.6$ with Altona.

203 Figure 2. Detection of SARS-CoV-2 by SeeGene RT-QPCR from swabs stored in UTM medium, in

204 the absence of RNA extraction. Each dot represents one reaction corresponding to one patient.

205 Results are plotted as bar graphs with standard deviation. ' $n$ ' stands for the number of positive

206 RT-QPCR detection.

208 Figure 3. Detection of SARS-CoV-2 from swabs stored in Hanks medium. Results are plotted as

209 bar graphs with standard deviation. Ct values from amplification of extracted RNA from swabs

210 stored in Hanks medium using Altona's kit (Altona, S gene) and Seegen's kit (RNA_N; N gene, 
211 following an additional freeze/thaw cycle), or directly from Swabs in Hanks medium (No_Extr_N;

212 N gene) using SeeGene's kit.

213

214 Figure 4. Detection of SARS-CoV-2 by SeeGene RT-QPCR from swabs stored in molecular water.

215 Results are plotted as bar graphs with standard deviation. Serial dilutions were performed in

216 water from a swab stored in water. Ct values from amplification of extracted RNA from swabs

217 stored in water medium using Altona's kit (Altona_RNA, S and E genes) and Seegen's kit

218 (SeeGene_RNA; N and E genes), or directly from Swabs in water medium (H20; N and E gene)

219 using SeeGene's kit. 'na' means 'not available'.

221 Figure 5. Detection of SARS-CoV-2 by SeeGene RT-QPCR from RNA from swabs diluted in saline

222 water. Results are plotted as bar graphs with standard deviation. One hundredth dilution of 5

223 samples with high Ct value were performed in molecular water and in saline water. Ct values

224 obtained from RNA eluates diluted in water medium (H2O) or in saline water (Saline) using

225 Seegen's kit (SeeGene_RNA; RDRP, N and E genes).

226

227 Figure 6. Stability of swabs (not extracted) stored in $\mathrm{H} 2 \mathrm{O}$ at $4^{\circ} \mathrm{C}$.

228 Serial dilutions were performed in molecular grade water from a swab stored in RNAse inhibitor

229 containing water. Ct values from direct amplification from swab and dilutions was measured

230 using Seegen's kit (SeeGene_RNA; RdRP, N and E genes) following storage for 0, 1, 3 and 5 days. 
TABLE 1- Number of positive samples detected using different methods

\begin{tabular}{|c|c|c|c|c|c|c|}
\hline & RNA Abott & RNA & $\mathrm{H} 2 \mathrm{O}$ & Saline & UTM & Hanks \\
\hline Assay 1 & $n=9$ & $n=9(100 \%)$ & & & $n=9(100 \%)$ & \\
\hline Assay 2a & $n=7$ & $n=7(100 \%)$ & & & & \\
\hline Assay $2 b$ & $n=30$ & $n=30(100 \%)$ & & & & $\mathrm{n}=24(80 \%)$ \\
\hline Assay 3a* & $n=8$ & $n=8(100 \%)$ & & & $n=8(100 \%)$ & \\
\hline Assay $3 b^{*}$ & $n=7$ & $n=6(83,3 \%)$ & $n=6(85,7 \%)^{\&}$ & & & \\
\hline Assay 4b & $n=5$ & $n=5(100 \%)$ & $n=5(100 \%)$ & $n=5(100 \%)$ & & \\
\hline Assay 4c & $n=64$ & & & & & $n=47(73,4 \%)$ \\
\hline
\end{tabular}

$(*)$ Results confirmed in different volumes of MasterMix

\& Missed positive sample ( $\mathrm{Ct}=35.6$ in Altona) was detected at repetition on SeeGene $\mathrm{Ct}=37.1$ from swab stored in $\mathrm{H} 2 \mathrm{O}$ but not from extracted RNA.

TABLE 2- Mean Ct values (targets with best concordance)

\begin{tabular}{|c|c|c|c|c|c|c|}
\hline & RNA Abott ${ }^{a}$ & RNA & $\mathrm{H} 2 \mathrm{O}$ & Saline & UTM & Hanks \\
\hline Assay 1 & $17,28 \pm 6,98^{\mathrm{a}}$ & $21,77 \pm 8,38$ & & & $24,22 \pm 3,52$ & \\
\hline Assay 2a & $30,65 \pm 4,32^{\mathrm{a}}$ & $31,9 \pm 4,63$ & & & & \\
\hline Assay 2b & $24,45 \pm 4,35^{\mathrm{a}}$ & $26,57 \pm 5,18$ & & & & $31,22 \pm 6,32$ \\
\hline Assay 3a* & $28,75 \pm 2,2^{\mathrm{a}}$ & $31,15 \pm 2,3$ & & & $30,16 \pm 4,6$ & \\
\hline Assay $3 b^{*}$ & $28,02 \pm 5,1^{a}$ & $30,73 \pm 5,14$ & $31,65 \pm 4,8$ & & & \\
\hline Assay 4b & $13,32 \pm 0,67^{\mathrm{a}}$ & & $22,6 \pm 0,7^{b}$ & $32,98 \pm 2,8^{b}$ & & \\
\hline Assay 4c & $21,28 \pm 5,67^{a}$ & & & & & $30,68 \pm 9,66$ \\
\hline
\end{tabular}

${ }^{a}$ Abott Ct values results were added for reference and should not be directly compared to SeeGene's: RT-QPCRs were performed following an additional freeze/thaw cycle for SeeGene, less RNA and different primers. ${ }^{\mathrm{b}}$ These samples originate from RNA eluates diluted hundred times. $\left({ }^{*}\right)$ Results confirmed in different volumes of MasterMix. 
Figure 1. Ranges of Cts detected from RNA extracted from patients Swabs.

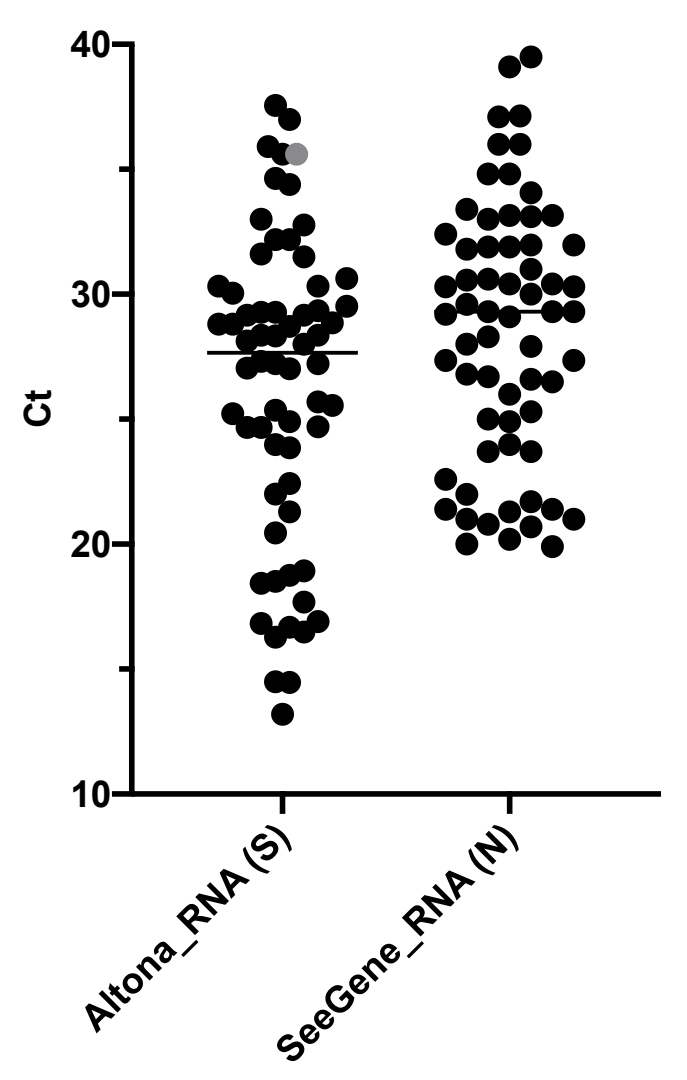

Each dot represents 1 of 65 reaction, each corresponding to a patient. The grey dot represents the only sample that was missed using SeeGene, detected at $\mathrm{Ct}=35.6$ with Altona. 
Figure 2. Detection of SARS-CoV-2 by SeeGene RT-QPCR from swabs stored in UTM medium, in the absence of RNA extraction.

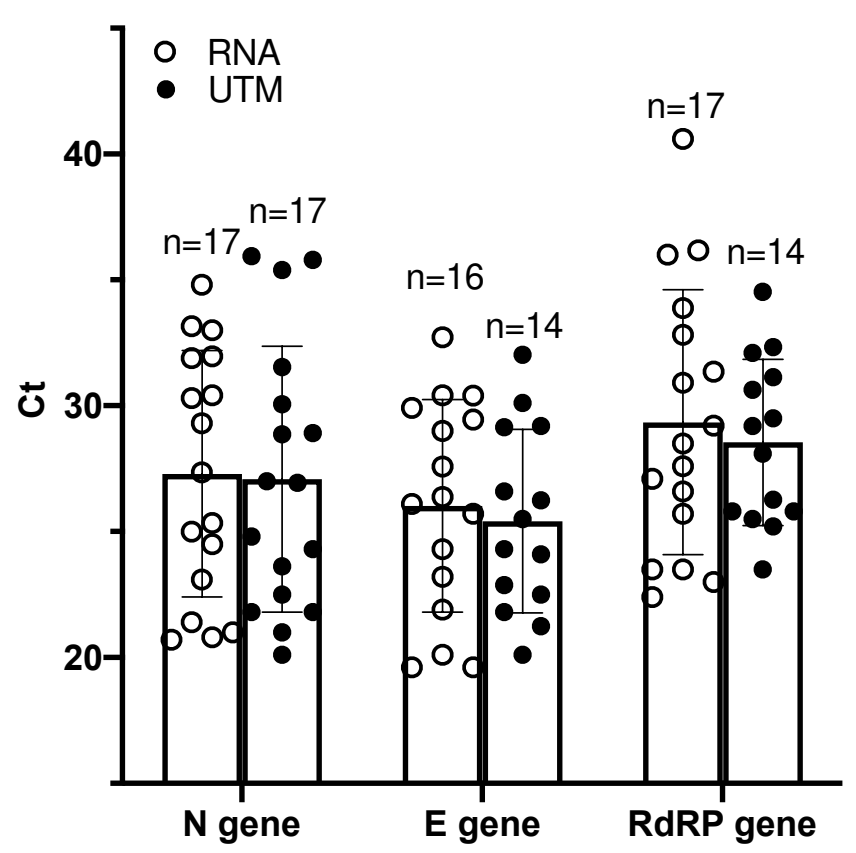

Each dot represents one reaction corresponding to one patient. Results are plotted as bar graphs with standard deviation. ' $n$ ' stands for the number of positive RT-QPCR detection. 
Figure 3. Detection of SARS-CoV-2 from swabs stored in Hanks medium.

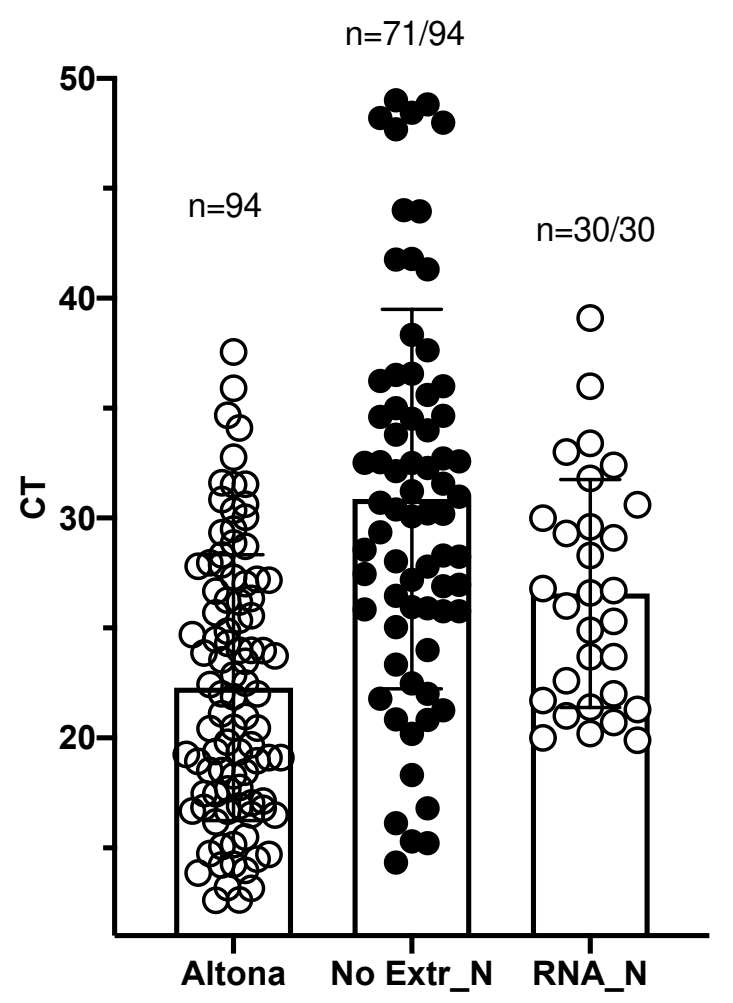

Results are plotted as bar graphs with standard deviation. Ct values from amplification of extracted RNA from swabs stored in Hanks medium using Altona's kit (Altona, S gene) and Seegen's kit (RNA_N; N gene, following an additional freeze/thaw cycle), or directly from Swabs in Hanks medium (No_Extr_N; N gene) using SeeGene's kit. 
Figure 4. Detection of SARS-CoV-2 by SeeGene RT-QPCR from swabs stored in molecular water.

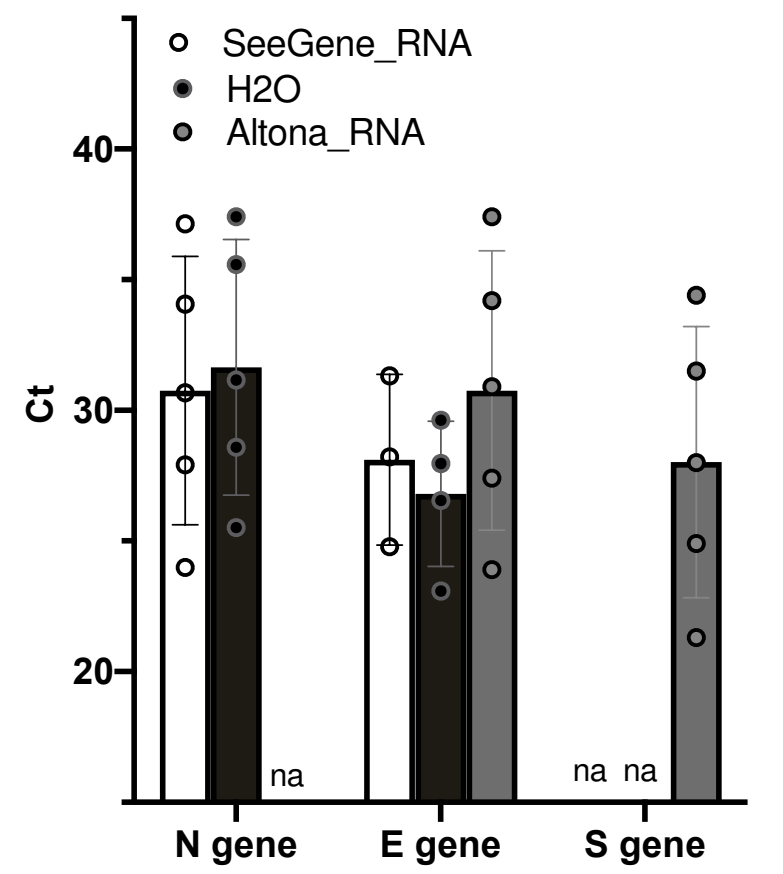

Results are plotted as bar graphs with standard deviation. Serial dilutions were performed in water from a swab stored in water. Ct values from amplification of extracted RNA from swabs stored in water medium using Altona's kit (Altona_RNA, S and E genes) and Seegen's kit (SeeGene_RNA; N and E genes), or directly from Swabs in water medium (H20; N and E gene) using SeeGene's kit. 'na' means 'not available'. 
Figure 5. Detection of SARS-CoV-2 by SeeGene RT-QPCR from RNA from swabs diluted in saline water.

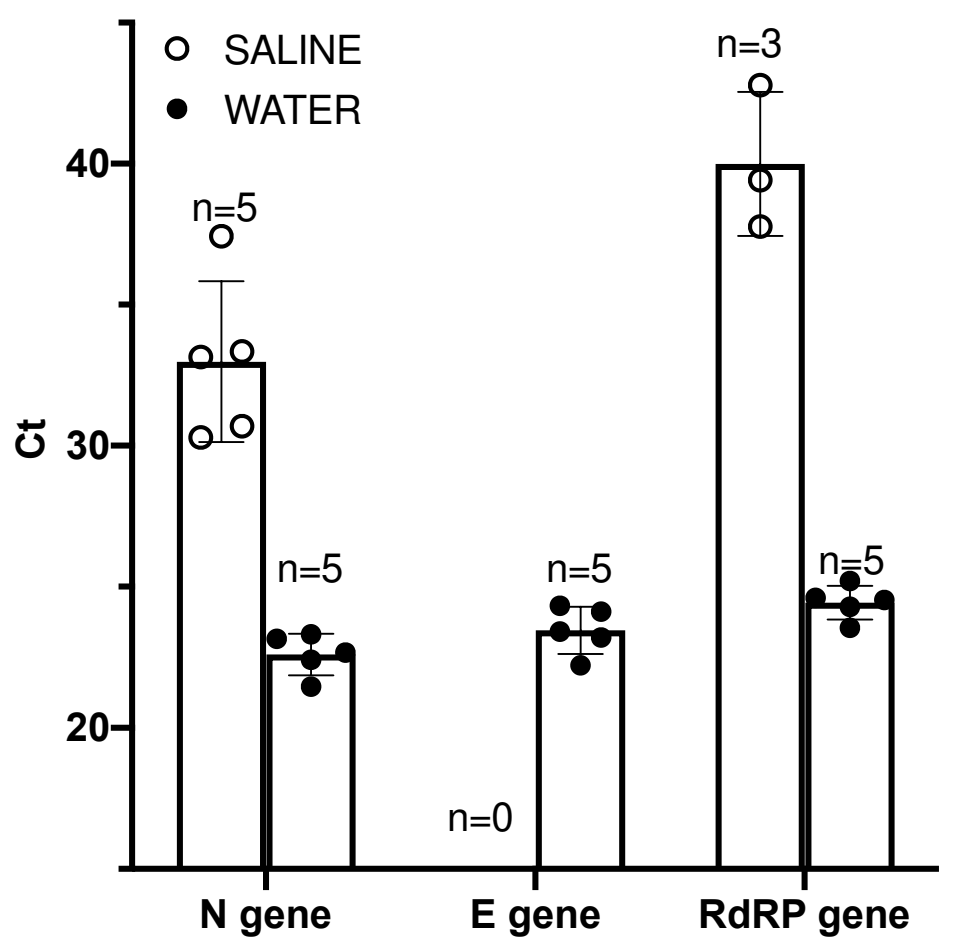

Results are plotted as bar graphs with standard deviations. One hundredth dilution of 5 samples with high $\mathrm{Ct}$ value were performed in molecular water and in saline water. $\mathrm{Ct}$ values obtained from RNA eluates diluted in water medium (H2O) or in saline water (Saline) using Seegen's kit (SeeGene_RNA; RDRP, N and E genes). 
Figure 6. Stability of swabs (not extracted) stored in molecular grade $\mathrm{H} 2 \mathrm{O}$ at $4{ }^{\circ} \mathrm{C}$.
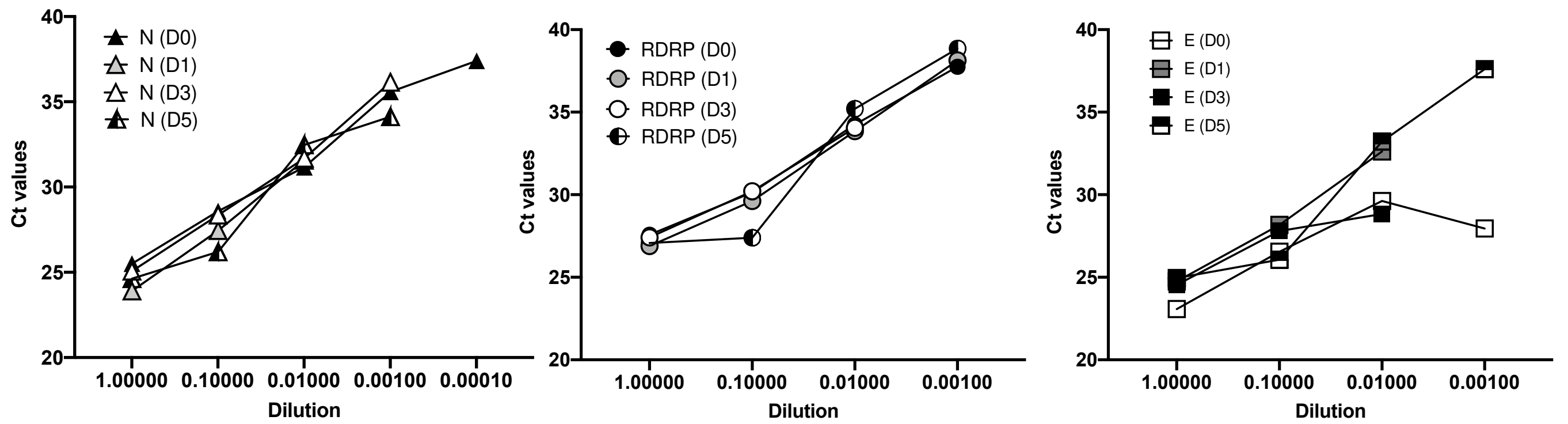

Serial dilutions were performed in molecular grade water from a swab stored in molecular grade water with RNAse inhibitor. Ct values from direct amplification from swab and dilutions was measured using Seegen's kit (SeeGene_RNA; RdRP, $\mathrm{N}$ and $\mathrm{E}$ genes) following storage for $0,1,3$ and 5 days. 